\title{
Valoración de la relación entre Rendimiento Académico y Condición Física en escolares zaragozanos
}

\section{Associations of Academic Performance and Physical Fitness in Zaragoza's adolescent students}

\author{
Castro López, R. .*, Pérez Gómez, V. ${ }^{2}$, Cachón Zagalaz, J. ${ }^{3}$ y Zagalaz Sánchez, Ma L. ${ }^{3}$ \\ 1 Universidad Internacional de la Rioja. \\ 2 Graduado en Ciencias de la Actividad Física y del Deporte. Máster Profesorado en Educación Secundaria modalidad Educación Física. \\ 3 Universidad de Jaén
}

\begin{abstract}
Resumen: Introducción: El estudio analiza las asociaciones entre la condición física y el rendimiento académico, atendiendo al género, curso y origen del alumnado.

Material y métodos: Fueron analizados 338 alumnos. El rendimiento escolar se basó en la nota media académica general, las notas de matemáticas y lengua, la media de estas dos materias y las asignaturas suspendidas. La Condición Física (CF) fue evaluada por la Batería EUROFIT. El tratamiento estadístico, situó la significación bilateral en .05.

Resultados: Aparece significación entre la CF y todas las variables académicas $(p<.01)$. La prueba con mayor significación es la flexibilidad (todas las variables académicas $p<.01$ ). Atendiendo al género, aparece en ambos y, basándonos en el curso, existe en los primeros cursos analizados. Respecto al origen, los alumnos españoles presentan significación siendo puntual en los alumnos inmigrantes.

Conclusiones: Se concluye la existencia de una relación entre la CF y el rendimiento escolar, variando en función de las pruebas y los elementos diferenciadores analizados.

Palabras clave: Rendimiento académico, Condición Física, adolescentes, género, curso, inmigrantes, nativos.
\end{abstract}

Abstract: Introduction: The aim of this study is to analyze the relationship between fitness and academic achievement based on variables such as gender, course and nationality.

Methods: 338 students were analyzed. Academic achievement was based on academic average, scores and average on mathematics and language and academic suspension. Fitness was evaluated with the EUROFIT Battery. The level of statistical significance was $p<0.05$.

Results: Significant results were observed in the total sample between fitness and academic performance $(p<.01)$. Flexibility test was significantly associated with academic achievement (all academic variables $p<.01$ ), In terms of gender, significant relationships appear in both. Depending on the course, significant relationships exist in the first courses. In terms of origin, native students present significant association but this association is punctual in foreign students.

Conclusions: This study shows a significant relationship between fitness and academic performance, depending on items and different characteristics presents in the study.

Key Words: academic performance, physical fitness, adolescents, gender, grade, immigrants, native.

\section{Introducción}

La relación entre la condición y aptitudes físicas y el rendimiento escolar ha sido y continúa siendo objeto de estudio. Una positiva relación entre estos dos parámetros, incrementaría la relevancia que tienen los profesionales de la EF en relación con el proceso de enseñanza-aprendizaje de nuestros escolares. Esta relación muestra diferencias en función de elementos diversificadores como la edad, género o IMC, entre otros, de los alumnos implicados en el estudio.

Atendiendo a la información previa, existen varios estudios que han analizado esa relación entre la CF de los escolares (Vergara, Elzel, Ramírez, Castro \& Cachón, 2015) y el rendimiento académico. A nivel internacional, uno de ellos que analizó a estudiantes estadounidenses de edades entre 8 y 18 años, encontró una relación significativa entre estas dos variables, siendo el mayor pico de esta asociación el comienzo

Dirección para correspondencia [Correspodence address]: Rosario Castro López. E-mail: charic110@hotmail.com de la Educación Secundaria (Van Dusen, Kelder, Kohl, Ranjit, \& Perry, 2011). Dentro de nuestras fronteras, también aparecen estudios relacionados con esta temática. Uno de ellos, realizado con estudiantes entre 9 y 11 años, afirma que el éxito académico se asociaba con los niveles más altos en cuanto a CF se refiere (Torrijos-Niño et al., 2014).

Analizando los elementos discriminantes que influyen en esta relación, el género aparece como un aspecto relevante. un estudio realizado con adolescentes suecos, observó en los chicos una relación entre el rendimiento escolar y la aptitud física, no siendo esto observado en las chicas (Kwak et al., 2009), sin embargo, en otro estudio realizado en USA, se observó una relación más fuertes en las chicas, que en los chicos (Grissom, 2005).

También, analizando la edad de los alumnos, se han observado artículos en los que esta relación no muestra diferencia en función del año de nacimiento (Sardinha, Marques, Martins, Palmeira, \& Minderico, 2014), aunque otro de ellos, 
muestra que esa relación aparece en edades jóvenes, siendo más débil a medida que el alumno avanza en edad (Kim et al., 2003).

Otra variable a examinar en esta relación, y dado que en nuestro país la densidad del alumnado inmigrante ha aumentado notablemente (Calero, Choi, \& Waisgrais, 2009; Pereda, de Prada, \& Actis, 2003), sería el origen de los mismos. Se han observado diferencias entre el rendimiento académico de este tipo de estudiantes en comparación con el alumnado autóctono, indicando como motivos de esta diferencia factores de adaptación, demográficos, familiares, etc. (Calero et al., 2009; Filigni, 1997; Pereda et al., 2003), no habiendo encontrado análisis que busquen esa diferencia en función de la CF, salvo el de Ruiz-Valdivia, Molero, Zagalaz \&Cachón, (2012) que buscó la opinión que el alumnado tiene de la asignatura de Educación Física (EF) y la influencia en el alumnado inmigrante.

Por todo ello, el objetivo del estudio será analizar si existe una relación significativa entre el rendimiento escolar y la CF en alumnos adolescentes, valorando esta relación según el género y la procedencia del alumnado.

\section{Material y método}

\section{Muestra}

Un total de 388 adolescentes sanos (media 14,86 1 ,3años), 189 chicos $(48,7 \%)$ y 199 chicas $(51,3 \%)$, han sido valorados. Los adolescentes cursan Educación Secundaria Obligatoria (ESO) y $1^{\circ}$ bachillerato, cursos en los que es obligatoria la EF, en un centro escolar sito en Zaragoza (España), siendo 334 $(86,1 \%)$ hijos de padres españoles y 54 (13,9\%) hijos de padre y/o madre inmigrante. El tipo de muestreo es no probabilístico intencional, en el que se usa como criterio de inclusión no tener adaptación curricular significativa.

\section{Instrumentos de valoración del rendimiento académico}

El rendimiento académico se analiza con cuatro variables: la nota académica, la nota de matemáticas y lengua castellana, la media de estas dos asignaturas y el número de suspensos. La nota académica es el promedio de las notas de todas las asignaturas cursadas por el adolescente en el segundo trimestre del curso. El valor de esta variable oscila entre 0 como nota mínima y 10 como nota máxima, redondeándola a un decimal. Para las pruebas estadísticas agrupamos a los participantes en tres conjuntos, el $1^{\circ}$, si la nota promedio es inferior a 5; el grupo $2^{\circ}$, si la nota se encuentra entre el 5 y el 7 , estando ambas calificaciones incluidas, y el $3^{\circ}$ si la nota promedio es superior a 7. La variable y la agrupación relativa a las notas de matemáticas y lengua, será similar a la indicada para la nota promedio, tanto de manera independiente la una de la otra, como cuando se valore la media de las dos asignaturas.

El número de suspensos es una variable entera entre 0 y el número total de asignaturas cursadas en ese ciclo de secundaria. En función del número de suspensos y atendiendo a la baremación de (Inglés et al., 2009), también se ha agrupado al alumnado en tres secciones, si tienen 3 o más suspensos, si tienen entre 1 y 2 asignaturas suspendidas (ambas inclusive), y si no tienen ninguna asignatura suspendida.

\section{Instrumentos de valoración de la Condición Física}

La CF se valora con seis capacidades: la velocidad de los miembros, la flexibilidad de tronco, la fuerza explosiva de tren inferior, la resistencia abdominal, la resistencia de brazos y hombros y la velocidad-coordinación. Para ello se realizaron seis test siguiendo las directrices de Adam, Renson, Klissouras, Ravazzolo, \& Tuxworth (1992) y de (Martínez, 2002): Plate Tapping, Sit and Reach test, salto de longitud, abdominales en 30 seg., test de suspensión de brazos y carrera de ida y vuelta a máxima velocidad.

Para la velocidad de los miembros se usa la prueba de Plate Tapping: El sujeto situado delante de la mesa, pone una mano en el centro de la placa rectangular. Con la otra (su mano hábil), tiene que tocar alternativamente los dos discos lo más rápido que pueda, pasando cada vez por encima de la mano colocada en el centro. Se deben realizar 25 ciclos de ida y vuelta. El test se realiza dos veces y se contabiliza el mejor resultado obtenido.

La Flexibilidad del tronco se mide con el "Sit and Reach test". Al inicio de la prueba el sujeto permanece sentado en el suelo con las piernas juntas y extendidas. El ejecutante está descalzo, con los pies pegados a la caja de medición y los brazos y manos extendidos (manteniendo una apoyada sobre la otra) y mirando hacia delante. A la seńal, el ejecutante flexiona el tronco adelante, empujando con ambas manos el cursor hasta conseguir la mayor distancia posible.

Para valorar la Fuerza explosiva del tren inferior se utiliza la prueba de salto de longitud a pies juntos: los participantes realizan un salto lo más lejos posible, sin caer atrás. Se valora el mejor de dos intentos. La mayor distancia horizontal fue medida en centímetros.

La Resistencia abdominal se valora con la prueba de abdominales en 30 segundos. El ejecutante se coloca tumbado en una colchoneta dura sobre la espalda, con las rodillas ligeramente flexionadas y de forma que las piernas queden abiertas a la anchura de los hombros. El movimiento consiste en una flexión del tronco tocando los codos sobre las rodillas, para volver a la posición de tumbados donde los hombros deben tocar con el suelo.

Para medir la resistencia de brazos y hombros se realiza el test de suspensión con flexión de brazos. El sujeto situándose debajo de la barra, con las manos en presa dorsal con una 
separación similar a la anchura de sus hombros, elevará su barbilla por encima de la barra (ayudado por el controlador). Debe intentar mantenerse en esta posición el mayor tiempo posible sin apoyarse en la barbilla. Se da por finalizado el test cuando sus ojos descienden por debajo de la barra.

La velocidad-coordinación se mide a partir de la Carrera de Ida y Vuelta (<<course navette >) a velocidad máxima. Situado de pie en la línea de salida, con un pie justo detrás de la línea. A la señal, tiene que correr lo más rápidamente posible hasta la otra línea, rebasarla con ambos pies y volver a velocidad máxima hasta la línea de salida. Esta fase constituye un ciclo. Tiene que efectuar 5 ciclos seguidos. El test se realizará una sola vez.

Para baremar las pruebas se han utilizado las escalas de percentiles de la Batería EUROFIT, según edad y género. Para este estudio se han tenido en cuenta las seis pruebas por separado y el promedio de ellas, llamando a éste valor "Promedio Condición Física (PCF)". Según este promedio, se han agrupado a los sujetos siguiendo las directrices de (Aguilar et al., 2011; Ortega et al., 2011; Ortega et al., 2005): grupo $1\left(<\mathrm{PCF}_{20}\right)$, grupo $2\left(\mathrm{PCF}_{20} \leq \mathrm{X}<\mathrm{PCF}_{40}\right)$, grupo $3\left(\mathrm{PCF}_{40} \leq \mathrm{X}<\mathrm{PCF}_{60}\right)$, grupo $4\left(\mathrm{PCF}_{60} \leq \mathrm{X}<\mathrm{PCF}_{80}\right)$ y grupo 5 $\left(\mathrm{X} \geq \mathrm{PCF}_{80}\right)$.

\section{Método estadístico}

El tratamiento de los datos recogidos se ha efectuado utilizando el paquete estadístico SPSS 20. Hemos aplicado una estadística descriptiva para hallar las medias, desviaciones estándar y la distribución en valores porcentuales de los niños según su género y procedencia.

Para comparar los datos entre dos grupos referidos al género, se utilizó una prueba $\mathrm{T}$ para muestras independientes. Las relaciones entre la CF, y los aspectos académicos, se realizaron mediante correlaciones bivariadas.

El nivel de significación fue del $5 \%(p<.05)$ para todos los análisis.

\section{Resultados}

Un total de 388 adolescentes sanos (189 chicos y 199 chicas) han sido incluidos en este estudio, siendo la edad media de los participantes de $14.86 \pm 1.3$ años. Un $13.9 \%$ de ellos son hijos de padre y/o madre inmigrante.

En cuanto a los valores académicos, la nota promedio de las chicas $(6.26 \pm 1.74)$ es significativamente mayor que la de los chicos $(5.77 \pm 1.6)(p=.005)$. Además, la de los alumnos españoles $(6.18 \pm 1.65)$ es significativamente mayor que la de los hijos de inmigrantes $(5.1 \pm 1.76)(p=.000)$.

El $52.8 \%$ de las chicas no han suspendido nada frente al $40.7 \%$ de chicos, y el $38.6 \%$ de los chicos suspenden 3 o más asignaturas frente al $21.6 \%$ de las chicas. El número de suspensos sí depende del género $(p=.019)$ y también de la procedencia ( $p=.000$ ) (tabla 1). Analizado las frecuencias, un $30.5 \%$ de los españoles obtiene una nota promedio superior al 7 , frente al $13 \%$ de alumnos de origen inmigrante, y la nota inferior a 5 , es de un $20.4 \%$ para los alumnos españoles frente a un $44.4 \%$ de los alumnos inmigrantes. En relación al número de suspensos, un $50 \%$ de los españoles no tienen ningún suspenso frente al $27.8 \%$ de los inmigrantes, y tres o más suspensos los obtienen un 26\% de los españoles frente a un $53.7 \%$ de los inmigrantes.

Entre las pruebas de CF realizadas utilizando la baremación oficial de la batería EUROFIT, no dependen ni del género ni de la procedencia de los alumnos, las pruebas que valoran la resistencia abdominal y la velocidad de los miembros superiores. Tampoco es dependiente del género del alumnado la prueba que valora la flexibilidad y de la procedencia del alumnado la que valora la resistencia de brazos y hombros. Sí que son dependientes del género del alumnado la prueba que valora la resistencia de brazos y hombros, siendo las chicas las que obtienen unos resultados significativamente superiores $(59.55 \pm 25.61)$ a la de los chicos $(50.35 \pm 27.87)(p=.001)$ (tabla 1), al igual que es dependiente de la procedencia del alumnado la prueba que valora la flexibilidad, siendo los alumnos españoles los que obtienen unos resultados significativamente superiores $(86.05 \pm 20.52)$ a la de los alumnos inmigrantes $(78.76 \pm 27.34)(p=.022)$ (tabla 1). Además, decir que las pruebas que valoran tanto la fuerza explosiva del tren inferior como la velocidad y coordinación son dependientes del género y procedencia.

En la prueba que valora la FE del tren inferior es superior los resultados de las chicas $(57.49 \pm 24.23)$ que los de los chicos (48.76 \pm 28.01$)(p=.001)$ (tabla 1), y los valores de los alumnos autóctonos $(54.46 \pm 26.15)$ que aquellos de origen inmigrante (45.63 \pm 27.38$)$ ( $p=.023$ ) (tabla 1). En la misma línea sucede con la prueba que valora la velocidad y coordinación, siendo más elevados los resultados obtenidos por las chicas $(76.79 \pm 20.26)$ que la de los varones $(71.70 \pm 25.22)(p=.029)$ (tabla 1) y la de los alumnos españoles $(75.23 \pm 22.28)$ que la de los estudiantes inmigrantes $(68.65 \pm 26.10)(p=.049)$ (tabla $1)$. 
Tabla 1. Valores descriptivos y relación estadística según sexo y procedencia.

\begin{tabular}{cccccccc}
\hline & Total & Varones & Mujeres & $p$ & Españoles & Inmigrantes & $p$ \\
\hline Edad (años) & $14.86 \pm 1.3$ & $14.92 \pm 1.2$ & $14.8 \pm 1.3$ & .390 & $14.85 \pm 1.28$ & $14.89 \pm 1.24$ & .849 \\
Nota Promedio & $6.03 \pm 1.7$ & $5.77 \pm 1.6$ & $6.26 \pm 1.74$ & $.005^{*}$ & $6.18 \pm 1.65$ & $5.10 \pm 1.76$ & $.000^{*}$ \\
Matemáticas & $5.28 \pm 2.3$ & $5.06 \pm 2.3$ & $5.48 \pm 2.2$ & .068 & $5.50 \pm 2.18$ & $3.91 \pm 2.29$ & $.000^{*}$ \\
Lengua Cast. & $5.9 \pm 2.4$ & $5.58 \pm 2.4$ & $6.2 \pm 2.4$ & $.011^{*}$ & $6.11 \pm 2.37$ & $4.63 \pm 2.53$ & $.000^{*}$ \\
Mat-Leng. & $5.59 \pm 2.2$ & $5.32 \pm 2.2$ & $5.85 \pm 2.5$ & $.013^{*}$ & $5.80 \pm 2.12$ & $4.27 \pm 2.23$ & $.000^{*}$ \\
No Suspensos & $1.91 \pm 2.6$ & $3.28 \pm 2.98$ & $2.18 \pm 2.73$ & $.019^{*}$ & $1.68 \pm 2.41$ & $3.30 \pm 3.00$ & $.000^{*}$ \\
Abdominales & $80.31 \pm 23.13$ & $81.28 \pm 24.14$ & $79.39 \pm 22.15$ & .423 & $81.09 \pm 22.75$ & $75.46 \pm 25.04$ & .097 \\
Longitud & $53.23 \pm 24.47$ & $48.76 \pm 28.01$ & $57.49 \pm 24.23$ & $.001^{*}$ & $54.46 \pm 26.15$ & $45.63 \pm 27.38$ & $.023^{*}$ \\
Brazos & $55.07 \pm 27.09$ & $50.35 \pm 27.87$ & $59.55 \pm 25.61$ & $.001^{*}$ & $55.03 \pm 26.66$ & $55.31 \pm 29.90$ & .944 \\
Velocidad-Coordinación & $74.31 \pm 22.93$ & $71.70 \pm 25.22$ & $76.79 \pm 20.26$ & $.029^{*}$ & $75.23 \pm 22.28$ & $68.65 \pm 26.10$ & $.050^{*}$ \\
Flexibilidad & $85.03 \pm 21.70$ & $84.23 \pm 22.68$ & $85.79 \pm 20.75$ & .479 & $86.05 \pm 20.52$ & $78.76 \pm 27.34$ & $.022^{*}$ \\
Plate Tapping & $70.18 \pm 24.56$ & $69.36 \pm 24.87$ & $70.95 \pm 24.30$ & .523 & $70.27 \pm 24.27$ & $69.63 \pm 26.53$ & .637 \\
Cond. Física & $69.69 \pm 16.65$ & $67.61 \pm 18.09$ & $71.66 \pm 14.94$ & $.016^{*}$ & $70.35 \pm 16.33$ & $65.57 \pm 18.12$ & $0.050^{*}$ \\
\hline
\end{tabular}

Si valoramos la relación entre el rendimiento académico y la CF total, así como las diferentes pruebas analizadas se observa una relación significativa para la totalidad de la muestra (tabla 2).

Si atendemos al sexo de los participantes, se observa que existe una significación entre la CF y los valores académicos para ambos sexos, a diferencia de la variable de matemáticas, en la que la relación es significativa para las mujeres $(p=.000)$, pero no para los varones ( $p=.2$ ) (tabla 2).

$\mathrm{Si}$ atendemos a la procedencia del alumnado, se observa una significación total en todas las variables académicas y la CF en los alumnos españoles ( $p=.000$ ) (Tabla 2). En el caso de los alumnos inmigrantes esa relación se vuelve menos significativa para los valores de lengua castellana $(p=.013)$, la media de matemáticas y lengua castellana $(p=.031)$ y para el número de suspensos ( $p=.13)$, no existiendo esta significación en la variable de matemáticas ( $p=.278)$ (tabla 2$)$.

Si valoramos la relación entre la CF y el rendimiento académico en función del curso en el que se encuentra matriculado el alumnado (tabla 3) aparecen discrepancias. Para los tres primeros cursos de la ESO, la relación entre la CF y el rendimiento escolar, es significativa para todas las variables académicas ( $p<.01)$ (tabla 3$)$. Para el cuarto curso de la ESO, la relación es significativa en la nota de matemáticas $(p<.01)$, la media de matemáticas y lengua castellana $(p<.05)$ y la nota promedio $(p<.05)$ (tabla 3). Para el último curso analizado, $1^{\circ}$ Bachillerato, esta relación no es significativa.

Tabla 2. Correlación de la Condición Física y el Rendimiento Académico según sexo y origen.

\begin{tabular}{lccccccccccc}
\hline Valores académicos & \multicolumn{10}{c}{ Condición Física } \\
& \multicolumn{2}{c}{ Total } & \multicolumn{1}{c}{ Varones } & \multicolumn{1}{c}{ Mujeres } & Españoles & \multicolumn{1}{c}{ Inmigrantes } \\
\cline { 2 - 12 } & Coef. Corr. & $p$ & Coef. Corr. & $p$ & Coef. Corr. & $p$ & Coef. Corr. & $p$ & Coef. Corr. & $p$ \\
\hline Nota Promedio & $.482^{* *}$ & .000 & $.388^{* *}$ & .000 & $.571^{* *}$ & .000 & $.484^{* *}$ & .000 & $.419^{* *}$ & .000 \\
Matemáticas & $.299^{* *}$ & .000 & $.219^{* *}$ & .2 & $.370^{* *}$ & .000 & $.308^{* *}$ & .000 & .150 & .278 \\
Lengua Cast. & $.477^{* *}$ & .000 & $.385^{* *}$ & .000 & $.496^{* *}$ & .000 & $.462^{* *}$ & .000 & $.336^{*}$ & .013 \\
Mat-Leng. & $.395^{* *}$ & .000 & $.314^{* *}$ & .000 & $.456^{* *}$ & .000 & $.413^{* *}$ & .000 & $.294^{*}$ & .031 \\
No Suspensos & $-.393^{* *}$ & .000 & $-.341^{* *}$ & .000 & $-.421^{* *}$ & .000 & $-.413^{* *}$ & .000 & $-.337^{*}$ & .013 \\
\hline
\end{tabular}

${ }^{*}$ Correlación $(p)$ significativa a .05

${ }^{* *}$ Correlación $(p)$ significativa a .01 
Tabla 3. Correlación de la Condición Física y el Rendimiento Académico según curso.

\begin{tabular}{lcccc}
\hline Valores académicos & \multicolumn{3}{c}{ Condición física } \\
\hline & $1^{\circ}$ E.S.O & $2^{\circ}$ E.S.O & $3^{\circ}$ E.S.O & 40E.S.O \\
Nota promedio & Coef. Corr. & Coef. Corr. & Coef. Corr. & Coef. Corr. \\
\cline { 2 - 5 } Matemáticas & $.572^{* *}$ & $.419^{* *}$ & $.494^{* *}$ & $.263^{*}$ \\
Lengua & $.439^{* *}$ & $.314^{* *}$ & $.300^{* *}$ & $.343^{* *}$ \\
Mat-Leng & $.644^{* *}$ & $.433^{* *}$ & $.414^{* *}$ & .098 \\
No suspensos & $.568^{* *}$ & $.399^{* *}$ & $.392^{* *}$ & .050 \\
\hline
\end{tabular}

*Significativo para valor inferior a .05

**Significativo para valor inferior a .01

\section{Discusión}

En primer lugar, en cuanto al rendimiento escolar, se ha observando que, las mujeres obtienen un mayor rendimiento académico que los hombres, siendo esto significativo en todas las variables, a excepción de la nota de matemáticas, coincidiendo con otros estudios internacionales (Kuhl \& Hannover, 2012; Weis, Heikamp, \& Trommsdorff, 2013), y nacionales (Barbero, Holgado, Vila, \& Chacón, 2007).

Continuando con esta variable de rendimiento académico, nuestro estudio muestra similitudes con otros estudios (Calero et al., 2009; Calero,\& Waisgrais, 2008), que han observado un menor rendimiento académico en aquellos alumnos de origen inmigrante con respecto al alumnado autóctono, pudiendo deberse esto al hecho de poseer este alumnado inmigrante un nivel socioeconómico inferior (Calero et al., 2009) hecho que se ha observado influyente a la hora de valorar el rendimiento académico (Anisef, Brown, Phythian, Sweet, \& Walters, 2010).

Atendiendo a la relación de este rendimiento académico y la CF, el análisis que hemos realizado ha mostrado una relación significativa para todas las variables, concordando con los obtenidos en estudios a nivel internacional que utilizando una batería de CF distinta a la del presente estudio, como es el FITNESGRAMM test battery (Coe, Pivarnik, Womack, Reeves, \& Malina, 2012); así como vario de ellos realizado en USA que encontró, que una mejora en los niveles de CF producía un beneficio en el rendimiento académico (Bezold et al., 2014; Chomitz et al., 2009). A nivel nacional, un estudio con niños entre los 9 y los 11 años, también encontró esta relación significativa (Torrijos-Niño et al., 2014).

Atendiendo individualmente a la relación entre las pruebas físicas y el rendimiento escolar, las pruebas de flexibilidad (todas las variables $p<.01$ ) y la prueba que valora la velocidad y coordinación (todas las variables $p<.01$, salvo matemáticas $p<.05)$, coincidiendo con estudios realizados fuera de nuestras fronteras que observaron una relación entre la prueba de flexi- bilidad y un elevado rendimiento en los baremos referidos a la memoria (Roebers, \& Kauer, 2009) y una relación entre aquellos valores más elevados de coordinación y velocidad de movimiento con el rendimiento académico (Nourbakhsh, 2006).

En el lado opuesto, aparecen la prueba que valora la fuerza explosiva, y la resistencia muscular de brazos y hombros, como las menos relevantes (lengua castellana $p<.01$ y la nota promedio $p<.05)$. Esta puntualidad en la relevancia concordaría con la disparidad en los resultados observados en diferentes estudios, ya que alguno de ellos que si han observado relación entre la resistencia muscular y los resultados académicos (EvelandSayers, Farley, Fuller, Morgan, \& Caputo, 2009), otros han encontrado que la fuerza muscular no está relacionada con el rendimiento académico (Esteban-Cornejo et al., 2014).

En función del género, se ha observado una correlación significativa en ambos sexos, siendo esta mayor en mujeres, (todas las variables $p<.01$ ), que en hombres (similar significación, salvo en matemáticas, la cual no existe).

Estos datos concordarían con un estudios realizados a nivel internacional con escolares tanto australianos (Dwyer, Sallis, Blizzard, Lazarus, \& Dean, 2001), como de los Estados Unidos (Bezold et al., 2014) que observó que un incremento de la CF tanto en chicos como en chicas producía una mejora en el rendimiento escolar.

Además, esa relación mayor en las mujeres que en los hombres también aparece en diferentes estudios con alumnos entre los 8 y los 11 ańos (Carlson et al., 2008; Eveland-Sayers et al., 2009).

En función del curso del alumnado, se observa que la relación entre el rendimiento escolar y la CF, es significativa en los tres primeros niveles educativos analizados (todas las variables del rendimiento académico $p<.01$ ), mientras que en $4^{\circ}$ E.S.O la significación es puntual (nota de matemáticas $p<.01$; la nota promedio y a media de matemáticas y lengua castellana $p<.05$ ), siendo inexistente en el último curso analizado (10 Bachillerato). Esto coincide con estudios internacionales que han observado que el pico en la asociación entre 
el rendimiento escolar y la CF se observa en las edades más jóvenes y en el comienzo de la Educación Secundaria (Kim et al., 2003; Van Dusen et al., 2011).

Para finalizar el estudio se ha analizado esta relación con los estudiantes de origen inmigrante. En este análisis se ha observado que para el alumnado nacional la significación con la CF es total relacionándolos con la CF, (todos los valores académi$\cos p<.01$ ), mientras que para los estudiantes inmigrantes, esta significación es menor (nota promedio $p<.01$; nota de lengua castellana, media de matemáticas y lengua castellana y número de suspensos $p<.05$ ). Esto concordaría con aquellos estudios que han encontrado diferencias en el rendimiento académico de este tipo de escolares, debido a motivos relacionados con un período de adaptación (Calero et al., 2009), adquisición de la lengua de uso escolar (Vila, 2006), influencia de las aspiraciones paternas hacia el estudio (Areepattamannil \& Lee, 2014), el nivel socioeconómico (Aktop, 2010; Lämmle, Worth, \& Bös, 2012), que afectan más a la población inmigrante que los posibles beneficios que se han observado que produce una buena CF con respeto al rendimiento académico.

\section{Conclusión}

Los resultados del presente estudio muestran que se han observado relaciones positivas significativas cuando nos re- ferimos al total de la CF con todas las variables tratadas del rendimiento académico. Valorando las diferentes pruebas de aptitud física, se han observado las mayores significaciones en la prueba de flexibilidad y la prueba que valora la velocidad y la coordinación. Las asociaciones entre las otras pruebas físicas y el rendimiento académico puntuales, siendo la resistencia de brazos y hombros y la fuerza explosiva del tren inferior las que menos significación muestran con el rendimiento académico. En función del género, se ha observado en ambos géneros una correlación positiva y significante entre la CF y los valores escolares, siendo algo superior en algunos parámetros en mujeres que en hombres. Atendiendo al curso escolar, se han encontrado relaciones significativas en $1^{\circ}, 2^{\circ}$ y $3^{\circ}$ ESO, puntuales en $4^{\circ} \mathrm{ESO}$ e inexistentes en $1^{\circ}$ Bachillerato.

Respecto al origen del alumnado, mientras que en los estudiantes españoles existe significación de la CF con todas las variables académicas analizadas, en el alumnado inmigrante esta significación es puntual.

Consideramos necesarios estudios futuros que relacionen este rendimiento académico y CF con variables relevantes como son las socioeconómicas complementando la valoración de la CF con métodos tanto subjetivos como objetivos que valoren la Actividad Física, en muestras mayores y suficientemente representativas de la población.

\section{Referencias Bibliográficas}

1. Adam, C., Renson, R., Klissouras, V., Ravazzolo, M., \& Tuxworth, W. (1992). Eurofit: Test Europeo de Aptitud Fisica. Madrid: Ministerio de Educación y Ciencia.

2. Aguilar, A. C., Pradilla, A., Mosquera, M., Gracia, A. B., Ortega, J. G., Leiva, J. H., \& Ramírez-Vélez, R. (2011). [Percentile values for physical condition for Cali, Colombian children and adolescents], Biomédica, 31(2), 242-249.

3. Aktop, A. (2010). Socioeconomic status, physical fitness, self-concept, attitude toward physical education, and academic achievement of children, Percept Mot Skills, 110(2), 531-546.

4. Anisef, P., Brown, R. S., Phythian, K., Sweet, R., \& Walters, D. (2010) Early school leaving among immigrants in Toronto secondary schools, Can Rev Sociol 47(2), 103-128.

5. Areepattamannil, S., \& Lee, D. H. (2014). Linking immigrant parents' educational expectations and aspirations to their children's school performance, J Genet Psychol, 175(1-2), 51-57.

6. Barbero, I., Holgado, F. P., Vila, E.,\& Chacón, S. (2007). Actitudes, hábitos de estudio y rendimiento en matemáticas: Diferencias por género, Psicothema, 19(3), 413-442.

7. Bezold, C. P., Konty, K. J., Day, S. E., Berger, M., Harr, L., Larkin, M., \&Stark, J. H. (2014). The effects of changes in physical fitness on academic performance among New York City youth, $J$ Adolesc Health 55(6), 774-781.

8. Calero, J., Choi, A.,\& Waisgrais, S. (2009). Determinantes del rendimiento educativo del alumnado de origen nacional e inmigrante, Cuadernos Económicos del ICE, 78, 281-311.

9. Calero, J., \& Waisgrais, S. (2008). ¿Qué determina el rendimiento de los alumnos inmigrantes? Una primera aproximación a partir de PISA06, Investigaciones de Economía de la Educación, 3, 499-508.
10. Carlson, S. A., Fulton, J. E., Lee, S. M., Maynard, L. M., Brown, D. R., Kohl, H. W., \& Dietz, W. H. (2008). Physical education and academic achievement in elementary school: data from the early childhood longitudinal study, Am J Public Health, 98(4), 721-727.

11. Chomitz, V. R., Slining, M. M., McGowan, R. J., Mitchell, S. E., Dawson, G. F., \& Hacker, K. A. (2009). Is there a relationship between physical fitness and academic achievement? Positive results from public school children in the northeastern United States, J Sch Health, 79(1), 30-37.

12. Coe, D. P., Pivarnik, J. M., Womack, C. J., Reeves, M. J., \& Malina, R. M. (2012). Health-related fitness and academic achievement in middle school students, J Sports Med Phys Fitness, 52(6), 654-660.

13. Dwyer, T., Sallis, J. F., Blizzard, L., Lazarus, R., \& Dean, K. (2001) Relation of Academic Performance to Physical Activity and Fitness in Children, Pedriatic Exercise Science, 13(3), 225-237.

14. Esteban-Cornejo, I., Tejero-González, C. M., Martinez-Gomez, D., del-Campo, J., González-Galo, A., Padilla-Moledo, C.,\&Veiga,O.L. (2014). Independent and combined influence of the components of physical fitness on academic performance in youth, J Pediatr, 165(2), 306-312.

15. Eveland-Sayers, B. M., Farley, R. S., Fuller, D. K., Morgan, D. W., \& Caputo, J. L. (2009). Physical fitness and academic achievement in elementary school children, J Phys Act Health, 6(1), 99-104.

16. Fuligni, A. J. (1997). The academic achievement of adolescents from immigrant families: the roles of family background, attitudes, and behavior, Child Dev, 68(2), 351-363.

17. Grissom, J. B. (2005). Physical fitness and academic achievement, Journal of Exercise Physiology Online, 8(1), 11-25.

18. Inglés, C.J., Benavides, G., Redondo, J., García-Fernández, J.M., Ruiz- 
Esteban C., Estévez C., \& Huescar, E. (2009). Conducta prosocial y rendimiento académico en estudiantes españoles de ESO, Anales psicologia, 25, 93-101.

19. Kim, H. Y., Frongillo, E. A., Han, S. S., Oh, S. Y., Kim, W. K., Jang, Y. A., \&Kim, S. H. (2003). Academic performance of Korean children is associated with dietary behaviours and physical status, Asia Pac J Clin Nutr, 12(2), 186-192.

20. Kuhl, P., \& Hannover, B. (2012). Differenzielle Benotungen von Madchen and Jungen: Deir Enfluss der von der Lehkraft eingeschäzten Kompetenz zum selbsgesteurten Lennen [Different grading for girls versus boys? Examing the impact of student'ability for self-regulated learning as perceived by their teacher], Zeitschrift für Entwicklungspsychologie und Päagogische Psychologie, 44, 153-162.

21. Kwak, L., Kremers, S. P., Bergman, P., Ruiz, J. R., Rizzo, N. S., \& Sjöström, M. (2009). Associations between physical activity, fitness, and academic achievement, J Pediatr, 155(6), 914-918.

22. Lämmle, L., Worth, A., \& Bös, K. (2012). Socio-demographic correlates of physical activity and physical fitness in German children and adolescents, Eur J Public Health, 22(6), 880-884.

23. Martínez López, E.J. (2002). Pruebas de Aptitud Física. Barcelona: Editorial Paidotribo.

24. Nourbakhsh, P. (2006). Perceptual-motor abilities and their relationships with academic performance of fifth grade pupils in comparison with Oseretsky scale, Kinesiology, 38(1), 40-48.

25. Ortega, F. B., Artero, E. G., Ruiz, J. R., Espańa-Romero, V., JiménezPavón, D., Vicente-Rodríguez, G., \&Castillo, M.J. (2011). Physical fitness levels among European adolescents: the HELENA study, $\mathrm{Br} \mathrm{J}$ Sports Med, 45(1), 20-29.

26. Ortega, F. B., Ruiz, J. R., Castillo, M. J., Moreno, L. A., GonzálezGross, M., Wärnberg, J.,\&Gutiérrez, A. (2005). [Low level of physi- cal fitness in Spanish adolescents. Relevance for future cardiovascular health (AVENA study)], Rev Esp Cardiol, 58(8), 898-909.

27. Pereda, C., de Prada, M. Á., \& Actis, W. (2003). Alumnas y alumnos de origen extranjero: distribución y trayectorias escolares diferenciadas, Cuadernos de pedagogía, 326, 63-68.

28. Roebers, C. M., \& Kauer, M. (2009). Motor and cognitive control in a normative sample of 7-year-olds, Dev Sci 12(1), 175-181.

29. Ruiz-Valdivia, M., Molero-López Barajas, D., Zagalaz-Sánchez, M. L., \& Cachón-Zagalaz (2012). Análisis de la integración del alumnado inmigrante a través de las clases de EF,Apunts. EF y Deportes, 108(2), 26-34.

30. Sardinha, L. B., Marques, A., Martins, S., Palmeira, A., \& Minderico, C. (2014). Fitness, fatness, and academic performance in seventh-grade elementary school students, BMC Pediatr, 14, 176

31. Torrijos-Niño, C., Martínez-Vizcaíno, V., Pardo-Guijarro, M. J., García-Prieto, J. C., Arias-Palencia, N. M., \& Sánchez-López, M. (2014). Physical Fitness, Obesity, and Academic Achievement in Schoolchildren, The Journal of pediatrics, 165(1), 104-109.

32. Van Dusen, D. P., Kelder, S. H., Kohl, H. W., Ranjit, N., \& Perry, C. L. (2011). Associations of physical fitness and academic performance among schoolchildren, $J$ Sch Health 81(12), 733-740.

33. Vergara, M., Elzel, L., Ramírez, R., Castro, R., \& Cachón, J. (2015). Actividad física en estudiantes de octavo curso de educación básica de la comuna de río bueno (Chile),Journal of Sport and Health Research, $7(1), 43-54$.

34. Vila, I. (2006). Language, school and inmigration, Cultura y educación, 18(2), 127-142.

35. Weis, M., Heikamp, T., \& Trommsdorff, G. (2013). Gender differences in school achievement: The role of self-regulation, Front Psychol, 4, 442 